\title{
CIRCUITO DE TREINAMENTO \\ E CAPACIDADES FISICAS EM IDOSOS: \\ UMA REVISÃO SISTEMÁTICA
}

\author{
Dayzi Raquel Ferreira Paiva ${ }^{1}$ \\ Paloma Katlheen Moura Melo² \\ Gerian Lopes do Nascimento ${ }^{3}$ \\ Jussara Cristina Alves Medeiros ${ }^{4}$ \\ Maria Irany Knackfuss 5
}

resumo

Introdução: Um dos grandes benefícios que o exercício físico promove no envelhecimento é a preservação das capacidades funcionais. ○ circuito de treinamento visa melhorar controle corporal, equilí-

\footnotetext{
1 Graduada em Educação Física. Pós-graduanda em Fisiologia do Exercício e Treinamento Funcional da Faculdade do Vale do Jaguaribe (FVJ). Personal Trainer. E-mail: dayzipaiva@gmail.com.

2 Graduada em Nutrição. Mestre em Saúde e Sociedade. Professora adjunta da Faculdade do Vale do Jaguaribe (FVJ) e Faculdade Nova Esperança de Mossoró. E-mail: palomakatheen@hotmail.com. 3 Graduado em Educação Física. Mestre em Saúde e Sociedade. Professor substituto da Universidade do Estado do Rio Grande do Norte (UERN) vinculado ao Departamento de Educação Física. E-mail: gerian.lopes@outlook.com.

4 Graduada em Nutrição. Mestre em Saúde e Sociedade. Nutricionista da Prefeitura Municipal de São Miguel/RN. E-mail: jussaracalves@gmail.com.

5 Graduada em Educação Física. Doutora em Ciências da Saúde. Professor titular da Universidade do Estado do Rio Grande do Norte (UERN) vinculado ao Departamento de Educação Física. E-mail: kmariairany@yahoo.com.br.
} 
brio muscular, diminuindo a incidência de lesões e aumentando a eficiência dos movimentos. O objetivo deste trabalho é apresentar as evidências cientificas disponíveis na literatura sobre a relação entre a aplicação do circuito de treinamento e capacidades físicas em idosos. Métodos: Os bancos de dados Bireme, Web of Science, PubMed e Science Direct foram utilizados para a seleção dos artigos publicados nos últimos cinco anos. Resultados: Apenas quatro estudos foram selecionados para a realização da revisão sistemática, preenchendo os critérios de inclusão e exclusão. Conclusão: Diante das evidências existentes em relação à intervenção baseada em circuitos de treinamento, foi observado melhora no funcionamento físico e fisiológico dos idosos.

palavras-chave

Aptidão Física. Treinamento em Circuito. Exercícios Baseados em Circuitos. Idosos.

\section{Introdução}

Um dos grandes benefícios que o exercício físico promove no envelhecimento é a preservação da capacidade funcional, promovendo maior manutenção dos sistemas fisiológicos, evitando seu rápido declínio (MIRANDA et al., 2016). Para uma efetiva prevenção, ou redução das perdas funcionais que estão associadas ao envelhecimento, se faz necessário à inclusão de um programa regular de exercícios físicos, por se tratar de uma terapia não medicamentosa eficiente que auxilia o controle e manutenção das mudanças decorrentes do processo de envelhecimento, e que promove independência e autonomia nas atividades do cotidiano (MIRANDA et al., 2016).

O circuito de treinamento visa melhorar a capacidade funcional, através de exercícios que estimulam os receptores proprioceptivos presentes no corpo, os quais proporcionam um desenvolvimento da consciência sinestésica e do controle corporal, equilíbrio muscular estático e dinâmico e, consequentemente, diminui a incidência de lesões e aumenta a eficiência dos movimentos (LEAL et al., 2009). Com isso, proporciona melhorias dos aspectos neurológicos, utilizando exercícios que desafiam e estimulam a adaptação do sistema nervoso (SILVA, 2011).

Embora existam algumas evidências que apontam que o circuito de treinamento produz efeitos benéficos, ainda são escassos os estudos de revisões 
sistemáticas que tentam analisar as relações que um circuito de treinamento interfere nas capacidades física de idosos, sendo essa de suma importância para o possível direcionamento de estratégias dos profissionais de saúde que trabalham com essa população. Assim, objetiva-se neste estudo, analisar as evidências científicas sobre a relação entre a aplicação do circuito de treinamento e capacidades físicas em idosos.

\section{Metodologia}

\subsection{Critérios de seleção}

Os delineamentos metodológicos deste estudo se caracterizaram por uma revisão sistemática, sendo definidos como descritores das palavras: Physical fitness, Circuit training, Circuit-Based Exercise e Elderly. Os termos incluídos para variação foram: "circuito de treinamento", "exercício em circuito", "idosos" e "pessoas mais velhas". Também foram adotados os operadores booleanos AND e OR na busca dos artigos. Desta forma, foram descritos nas bases: Physical fitness AND circuit training AND elderly, Circuit-based exercise AND physical fitness AND elderly, Physical fitness AND circuit-based exercise OR circuit training AND elderly e Circuit training AND physical fitness AND elderly. Foi realizada uma leitura de todos os títulos e, após, foram selecionados os resumos e os artigos que restaram foram lidos na íntegra até selecionar os artigos para realizar a revisão sistemática. Os artigos utilizados para a pesquisa foram publicados de 2012 a 2016.

Sendo incluídos: 1) estudos com intervenção; 2) idosos de ambos os sexos; 3) artigos publicados em português, inglês e espanhol. Foram excluídos na análise: 1) Estudos que analisaram apenas exercício físico com outro tipo de população; 2) estudos com animais irracionais; 3) artigos pagos.

\subsection{Estratégias de banco de dados e pesquisa}

Esta pesquisa foi conduzida por meio de levantamento em banco de dados: Bireme, Web of Science, PubMed e Science Direct, no período de agosto a setembro de 2017. A estratégia da pesquisa foi baseada em uma combinação de dois parâmetros de pesquisa: circuito de treinamento e capacidades físicas em idosos.

Na primeira fase, 3110 artigos foram localizados. Após filtrar pela data de publicação, restaram apenas 696 artigos nas seguintes bases de dados: PubMed $\mathrm{n}=98$, Science Direct $\mathrm{n}=555$, Bireme $\mathrm{n}=23$, Web of Science $\mathrm{n}=20$, conforme descrito na figura 1. 


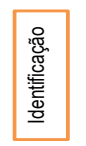

696 Scientific Articles PubMed (98)

Science direct $(555)$

Bireme (23)

Web of Science (20)
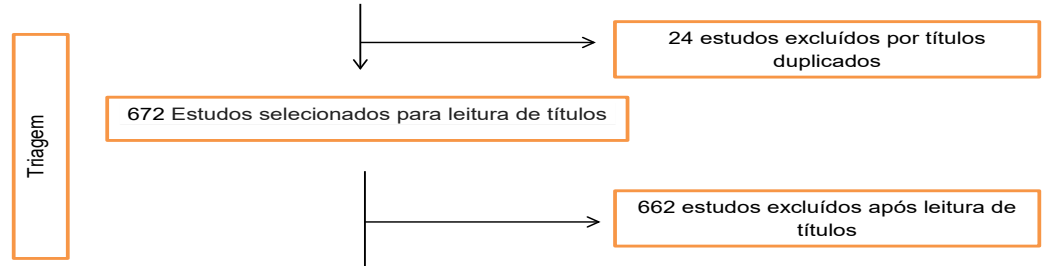

672 Estudos selecionados para leitura de títulos

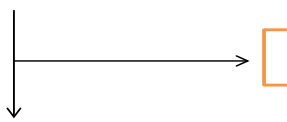

662 estudos excluídos após leitura de títulos

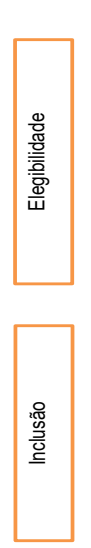

10 estudos selecionados para leitura de resumos

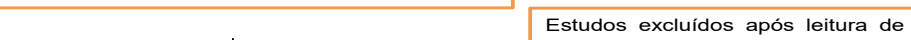
resumo:

- Retratava as competências da medicina. $(n=1)$

- Qualidade de vida após cirurgia. $(n=1)$

8 estudos selecionados para a leitura dos artigos na íntegra

Fonte: Elaborado pelas autoras.

Estudos excluídos após leitura do texto completo:

- Não relacionavam as variáveis estudadas. $(n=3)$

- Artigos pagos. $(n=2)$

Artigos incluídos na revisão sistemática. $(n=4)$

A avaliação de qualidade dos estudos foi realizada pelo escore Downs e Black e, como referência, o check-list preferido Itens para revisões sistemáticas e meta-análises (PRISMA) Relatórios. 
Foram identificados 3110 estudos dos quais restaram 696 após o filtro por data de publicação, sendo excluídas 24 duplicatas pelo Software EndNot X8.1, restando 672 artigos para a leitura dos títulos. Após leitura dos títulos, foram excluídos 662 artigos por não corresponderem as variáveis exigidas. Restando 10 artigos para a leitura dos resumos.

Após a leitura dos 10 resumos, foram excluídos dois artigos por retratarem as competências da medicina em geral e qualidade de vida após a realização da cirurgia bariátrica. Totalizando oito artigos para a leitura na integra. Após, três artigos foram excluídos por não atenderam aos critérios, dois eram artigos pagos e um por ser meta-análise, um artigo foi incluído das referências, restando quatro artigos para serem avaliados.

O Quadro 1 identifica que dois artigos foram realizados na Espanha e dois em Brasília, entre 2012 e 2016. A média das amostras variava entre um e 32 participantes, com 69,92 anos, maioria dos estudos eram experimentais, analisados por dinamômetros isocinéticos, que tem por objetivo aferir a força através do pico de torque isocinético. A pontuação composta fisicamente $\mathrm{e}$ mentalmente foram documentadas através do SF-12 versão 2 e a escala de equilíbrio de Berg avaliou o equilíbrio funcional em mulheres idosas (COSTA et al., 2012; GINÉ-GARRIGA et al., 2013; ARENAS et al., 2013; AVELAR et al., 2016).

Quadro 1 - Características sociodemográficos dos estudos avaliados $(n=4)$.

\begin{tabular}{|l|l|l|l|l|l|}
\hline Autor/Ano & País & Amostra & Idade & Design & Instrumentos \\
\hline $\begin{array}{l}\text { AVELAR et al., } \\
2016\end{array}$ & Brasil & $10 \mathrm{~F}$ & $68,3 \pm 7,35$ & $\begin{array}{l}\text { Quase- } \\
\text {-Experimental }\end{array}$ & $\begin{array}{l}\text { Dinamômetro } \\
\text { Isocinético }\end{array}$ \\
\hline $\begin{array}{l}\text { GINÉ-GARRIDA } \\
\text { et al., 2013 }\end{array}$ & Espanha & $26(\mathrm{~S}=\mathrm{NI})$ & $83,9 \pm 2,8$ & Experimental & $\begin{array}{l}\text { SF-12 } \\
\text { Versão 2) }\end{array}$ \\
\hline $\begin{array}{l}\text { ARENAS et al., } \\
\text { 2013 }\end{array}$ & Espanha & $16(\mathrm{~S}=\mathrm{NI})$ & $61,6 \pm 5,3$ & Experimental & $\begin{array}{l}\text { Dinamômetro } \\
\text { Isocinético }\end{array}$ \\
\hline $\begin{array}{l}\text { COSTA et al., } \\
\text { 2012 }\end{array}$ & Brasil & $32 \mathrm{~F}$ & $\begin{array}{l}65,61 \pm \\
4,72\end{array}$ & Experimental & $\begin{array}{l}\text { Escala de equilí- } \\
\text { brio de Berg }\end{array}$ \\
\hline
\end{tabular}

Fonte: Elaborado pelas autoras.

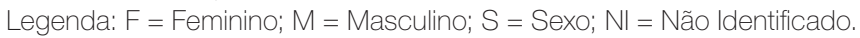

O Quadro 2 descreve as informações de caracterização das amostras dos estudos envolvidos nesta revisão, os tipos de intervenções realizadas, os procedimentos, resultados, conclusão e pontuação. A análise estatística usada pelos autores para a comparação de médias foram ANOVA, para examinar 
diferenças entre os grupos antes e após a intervenção, teste $t$ independente e testes não paramétricos. Todas as pontuações obtidas foram fundamentadas pela a escala Downs e Black alcançando valores acima de 20 pontos dos 27 itens validados.

Quadro 2 - Análise estatística, resultados e conclusões dos estudos incluídos ( $n=4)$.

\begin{tabular}{|c|c|c|c|c|c|c|}
\hline $\begin{array}{l}\text { Autor, } \\
\text { ano }\end{array}$ & $\begin{array}{l}\text { Tempo de } \\
\text { intervenção }\end{array}$ & $\begin{array}{l}\text { Tipo de } \\
\text { intervenção }\end{array}$ & $\begin{array}{l}\text { Análise } \\
\text { estatística }\end{array}$ & Resultados & Conclusão & $P$ \\
\hline $\begin{array}{l}\text { AVELAR et } \\
a l ., 2016\end{array}$ & 12 semanas & $\begin{array}{l}50 \text { ' de inter- } \\
\text { venção, 02X por } \\
\text { semana. 10' de } \\
\text { aquecimento, 30' } \\
\text { de exercícios de } \\
\text { equilíbrio e 10' de } \\
\text { repouso. Perma- } \\
\text { necendo 02' em } \\
\text { cada estação. }\end{array}$ & Anova & $\begin{array}{l}\text { Melhor } \\
\text { equilíbrio } \\
\text { estático } \\
\text { após o } \\
\text { circuito de } \\
\text { exercí- } \\
\text { cios de } \\
\text { equilíbrio. }\end{array}$ & $\begin{array}{l}\text { A intervenção } \\
\text { foi eficaz e } \\
\text { mostrou- } \\
\text {-se como } \\
\text { melhoras } \\
\text { importantes } \\
\text { para funciona- } \\
\text { mento físico. }\end{array}$ & 26 \\
\hline $\begin{array}{l}\text { GINÉ- } \\
\text {-GARRIDA } \\
\text { et al., } \\
2013\end{array}$ & 12 semanas & $\begin{array}{l}\text { Exercícios 02X } \\
\text { por semana. } \\
\text { Caminhada de } \\
\text { 10' e 5' pausa. } \\
\text { Variações de } \\
\text { velocidade, subir } \\
\text { em cadeiras, } \\
\text { escadas e } \\
\text { extensão de } \\
\text { pernas, pantur- } \\
\text { rilha e abdominal, } \\
\text { sem pesos } \\
\text { estabelecidos. }\end{array}$ & $\begin{array}{l}\text { Teste } t \\
\text { indepen- } \\
\text { dente }\end{array}$ & $\begin{array}{l}\text { Melhorias } \\
\text { no autor- } \\
\text { relato de } \\
\text { quedas e } \\
\text { as melho- } \\
\text { rias foram } \\
\text { mantidas } \\
\text { no } \\
\text { acompa- } \\
\text { nhamento } \\
\text { da semana } \\
26 \text {. }\end{array}$ & $\begin{array}{l}\text { O programa } \\
\text { foi valido e } \\
\text { a sustenta- } \\
\text { bilidade do } \\
\text { medo de } \\
\text { cair cumpre } \\
\text { os critérios } \\
\text { dentro da } \\
\text { intervenção. }\end{array}$ & 25 \\
\hline $\begin{array}{l}\text { ARENAS } \\
\text { et al., } \\
2013\end{array}$ & 12 semanas & $\begin{array}{l}\text { Intervenção } \\
\text { ocorreu 02X } \\
\text { por semana. } \\
\text { Aquecimento, } \\
\text { dois circuitos } \\
\text { e descanso de } \\
5^{\prime} .1^{\circ} \text { curvas de } \\
\text { pernas, levanta- } \\
\text { mento de pantur- } \\
\text { rilha sentado. } \\
2^{\circ} \text { extensão de } \\
\text { pernas e exercí- } \\
\text { cios de curvatura } \\
\text { e descanso de } \\
35^{\prime \prime} \text {. }\end{array}$ & Anova & $\begin{array}{l}\text { Foram } \\
\text { encon- } \\
\text { tradas } \\
\text { diferenças } \\
\text { significa- } \\
\text { tivas no } \\
\text { início do } \\
\text { treinamento } \\
\text { e não } \\
\text { observadas } \\
\text { diferenças } \\
\text { na confor- } \\
\text { midade do } \\
\text { treinamento. }\end{array}$ & $\begin{array}{l}\text { Os idosos } \\
\text { desta prática } \\
\text { obtiveram } \\
\text { ganhos de } \\
\text { força, massa } \\
\text { magra e } \\
\text { densidade } \\
\text { mineral óssea. } \\
\text { Prevenindo } \\
\text { doenças e } \\
\text { melhorando os } \\
\text { movimentos. }\end{array}$ & 23 \\
\hline
\end{tabular}




\begin{tabular}{|c|c|c|c|c|c|c|}
\hline $\begin{array}{l}\text { Autor, } \\
\text { ano }\end{array}$ & $\begin{array}{l}\text { Tempo de } \\
\text { intervenção }\end{array}$ & $\begin{array}{l}\text { Tipo de } \\
\text { intervenção }\end{array}$ & $\begin{array}{l}\text { Análise } \\
\text { estatística }\end{array}$ & Resultados & Conclusão & $\mathrm{P}$ \\
\hline $\begin{array}{l}\text { COSTA et } \\
\text { al., } 2012\end{array}$ & 13 semanas & $\begin{array}{l}\text { Exercícios 02X } \\
\text { por semana. 50' } \\
\text { de exercícios } \\
\text { multifatoriais. } 10 \text { ' } \\
\text { de aquecimento, } \\
35^{\prime} \text { de exercícios } \\
\text { sensórias e 5' de } \\
\text { volta à calma. }\end{array}$ & $\begin{array}{l}\text { Teste de } \\
\text { Wilcoxon }\end{array}$ & $\begin{array}{l}\text { Melhoras } \\
\text { significan- } \\
\text { temente no } \\
\text { equilíbrio } \\
\text { funcional } \\
\text { nos pós } \\
\text { testes. } \\
\text { Diminuindo } \\
\text { o índice de } \\
\text { possibili- } \\
\text { dades de } \\
\text { quedas. }\end{array}$ & $\begin{array}{l}\text { A participação } \\
\text { regular no } \\
\text { programa } \\
\text { foi capaz de } \\
\text { promover } \\
\text { o equilibrio } \\
\text { funcional } \\
\text { em idosas } \\
\text { e reduziu a } \\
\text { possibilidade } \\
\text { de quedas. }\end{array}$ & 24 \\
\hline
\end{tabular}

Fonte: Elaborado pelas autoras.

Legenda: $\mathrm{P}=$ Pontos da qualidade; $\mathrm{X}=$ Vezes.

\section{Discussão}

Com base na literatura analisada, compreende-se a preocupação em proporcionar um envelhecimento ativo e bem-sucedido, visando diminuir as diversas modificações do processo de envelhecimento em relação às alterações psicológicas, biológicas e funcionais, imitando a capacidade de realizar atividades da vida diária, tornando-os mais susceptíveis a doenças (FARIAS et al., 2015).

O circuito de treinamento é possível como uma forma de prevenção dos declínios dos desempenhos físicos podendo ser minimizado por meio desta prática, promovendo melhorias em aspectos importantes do funcionamento físico e fisiológico, tais como: equilíbrio estático e dinâmico, reduzindo os números de quedas, ganho de força e diminuição de doenças cardiovasculares (SOUZA JUNIOR et al., 2015).

Desta forma, podemos destacar que o principal item dos estudos dessa revisão foi que a participação nas intervenções promoveu independência funcional, reduziu o medo de cair, promoveu melhorias no equilíbrio, na velocidade da marcha, diminuiu o número de quedas e melhorou a qualidade de vida autorrelatada da vida dos idosos (COSTA et al., 2012; GINÉ-GARRIGA et al., 2013; ARENAS et al., 2013; AVELAR et al., 2016).

Algumas das principais perdas fisiológicas durante o processo de envelhecimento é a diminuição da força e, consequentemente, a perda e atrofia de fibras musculares. A importância de atividades físicas tem, em sua finalidade, promover efeitos benéficos a partir de práticas de circuitos de treinamento que, desta forma, melhora os aspectos funcionais e qualidade de vida de indivíduos mais velhos (MANDY et al., 2013). 
Alterações fisiológicas se instalam ao longo dos anos e podem ocasionar a diminuição das reservas fisiológicas e o aumento de marcadores biológicos, produzindo alterações intrínsecas (sistema cardiovascular, metabólica, condução fibra/muscular) e extrínsecas (alteração no sistema musculoesquelético, declínio na capacidade funcional, síndrome de imobilidade), desencadeando a vulnerabilidade do indivíduo. A inatividade física pode resultar em enfraquecimento muscular e ósseo, articulações enrijecidas, alterações nas vísceras causadas por elementos glandulares do tecido conjuntivo, aumento da pressão arterial e atrofia secundária, como a perda de peso (NETTO, 2004).

Assim, os estudos analisados nesta revisão associam a relação entre circuito de treinamento e capacidades físicas em idosos proporcionando a funcionalidade dos idosos. Com isso, é notória a importância da realização de novos estudos, pois poderão guiar profissionais da saúde na elaboração de estratégias norteadoras. Assim como, incentivar a importância inserção da atividade física nas suas diferentes formas e metodologias de circuito a fim de gerar impacto positivo na saúde e na qualidade de vida daqueles que envelhecem.

\section{Conclusão}

É sabido que a prática regular de atividade física pode reduzir os declínios funcionais, proporcionando a diminuição do enfraquecimento do tônus muscular, da estrutura óssea, do número de quedas e de doenças, melhorando, assim, as capacidades físicas dos idosos. Diante das evidências existentes em relação à intervenção baseada em circuitos, o circuito de treinamento melhora funcionamento físico e fisiológico dos idosos.

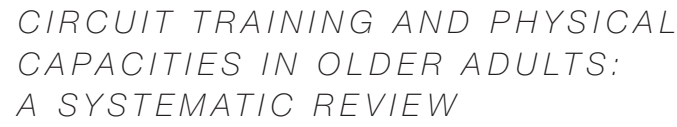

abstract

Introduction: One of the great benefits that physical activity promotes in ageing is the preservation of functional capabilities. The training circuit aims to improve body control, muscle balance, reducing the incidence of injuries and increasing the efficiency of movements. The objective of this work is to present the scientific evidence available in the literature on the relationship between the application of the training circuit and 

articles published in the last five years. Results: Only four studies were included to perform the systematic review, fulfilling the inclusion and exclusion criteria. Conclusion: Regular physical exercise can reduce functional declines by improving physical abilities in older adults. Given the existing evidence regarding circuit-based intervention, the training circuit improves physical and physiological functioning of older adults.

keywords

Physical Fitness. Circuit Training. Circuit-Based Exercises. Older Adults.

\section{referências}

ARENAS, S. et al. Effects of high-resistance circuit training in an elderly population. Experimental Gerontology, United States, v. 48, n. 3, p. 334-340, mar. 2013.

AVELAR, B. et al. Balance Exercises Circuit improves muscle strength, balance, and functional performance in older women. American Aging Association, United States, v. 38, p. 14, fev. 2016.

COSTA, J. et al. Efeitos do circuito de equilíbrio sobre o equilíbrio funcional e a possibilidade de quedas em idosas. Motricidade, Brasília, DF, v. 8, p. 485, mar. 2012. Supl. 2.

FARIAS, J. P. et al. Efeito de oito semanas de treinamento funcional sobre a composição corporal e aptidão física de idosos. Revista do Departamento de Educação Física, Santa Cruz, v. 16, n. 3, p. 194-197, jun. 2015.

GINÉ-GARRIGA, M. et al. The effect of functional circuit training on self-reported fear of falling and health status in a group of physically frail older individuals: a randomized controlled trial. Aging Clinical and Experimental Research, Suíça, v. 25, n. 3, p. 329--336, jun. 2013.

LEAL, S. et al. Efeitos do treinamento funcional na autonomia funcional, equilíbrio e qualidade de vida de idosas. Revista Brasileira de Ciência e Movimento, Brasília, DF, v. 17, n. 3, p. 61-69, out. 2009

MANDY, L. G. et al. Aging, functional capacity and eccentric exercise training. Aging and Disease, United States, v. 4, n. 6, p. 351-363, set. 2013.

MIRANDA, L. et al. Efeitos de 9 semanas de treinamento funcional sobre índices de aptidão muscular de idosas. Revista Brasileira de Prescrição e Fisiologia do Exercício, São Paulo, v. 10, n. 59, p. 386-394, jun. 2016

NETTO, F. Aspectos biológicos e fisiológicos do envelhecimento humano e suas implicações na saúde do idoso. Pensar a Prática, Goiânia, v. 7, n. 2, p. 75-84, mar. 2004.

SILVA, L. Treinamento Funcional Resistido e seus aspectos motivacionais em alunos de personal training. 2011. 63 f. Trabalho de Conclusão de Curso (Bacharelado em Educação Física) - Universidade Federal do Rio Grande do Sul, Porto Alegre, 2011.

SOUZA JUNIOR, S. et al. Força de membros superiores e inferiores de idosas praticantes e não praticantes de ginástica funcional. Saúde, Santa Maria, v. 41, p. 255-262, jun. 2015. 